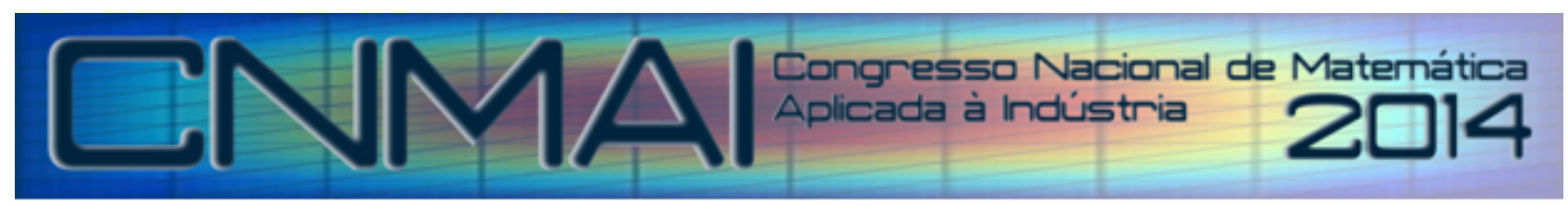

18 a 21 de novembro de 2014, Caldas Novas - Goiás

\title{
OTIMIZAÇÃO MULTIOBJETIVO E MULTIRESTRIÇÃO DA COBERTURA DE REDES DE FREQUÊNCIA ÚNICA
}

\author{
Adson Silva Rocha, adsonrocha@gmail.com ${ }^{1}$ \\ Leonardo da Cunha Brito, brito. lc@gmail. com $^{2}$ \\ Ciro José Almeida Macedo, ciro.macedo@ifg.edu.br ${ }^{3}$ \\ ${ }^{1}$ IF Goiano - Instituto Federal Goiano - Rua 88, no 310, Setor Sul, Goiânia - GO \\ ${ }^{2}$ UFG - Escola de Eng. Elétrica, Mecânica e de Computação - Av. Universitária, Setor Lest. Universitário, Goiânia-GO \\ ${ }^{3}$ IFG - Instituto Federal de Educação Ciências e Tecnologia de Goiás - Quartel do XX, Centro, Goiás-GO \\ Resumo. Esse artigo apresenta uma abordagem multiobjetivo e multirestrição para a otimização de sistemas de trans- \\ missão de vídeo digital, baseados em Redes de Frequência Única. O método proposto é capaz de prover curvas de \\ compromisso para a cobertura, custos e interferências, entre outros como nível de exposição à radiação. O método de \\ otimização de cobertura geral proposto pode ser estendido a outras redes sem fio, como sistemas de celular.
}

Palavras-chave: Orthogonal frequency-division multiplexing, Redes de Frequência Única, otimização multiobjetivo e multirestrição.

\section{INTRODUÇÃO}

A otimização de Redes de Frequência Única (RFU) (Rebhan et al., 1989; Vélez et al., 2000; Plets et al., 2010; Koutitas., 2010; Lanza et al., 2012, 2011; Ligeti et al., 1999; Ligeti, 1999; Küchen et al., 1996; Santella et al., 2004; Ricny., 2007) para os propósitos de transmissão de sinais de televisão digital geralmente emprega um modelo de otimização com um conjunto de variáveis de decisão, bem como um conjunto de métricas de performance, representados por funçõesobjetivo. Na literatura, diversos métodos de otimização de RFU tem sido reportados (Koutitas., 2010; Lanza et al., 2012, 2011; Ligeti et al., 1999; Ligeti, 1999; Küchen et al., 1996; Santella et al., 2004). Estes, independentemente do número de metas, aplicam o chamado método de somas ponderadas (Deb, 2001; Miettinen, 1999) para avaliar as soluções candidatas, nos quais a aptidão é dada pela soma ponderada das funções objetivo. Este modelo apresenta desvantagens, como por exemplo, a incapacidade de gerar todas as relações de compromisso entre as soluções em problemas nãoconvexos, a escolha dos pesos é realizada levando-se em conta apenas os fatores de ponderação desprezando a importância dos objetivos e a consistência dimensional na determinação dos mesmos. Além disso, existem várias soluções para um conjunto específico de pesos, ou seja, os pesos são também parâmetros a serem otimizados, o que pode resultar em desperdício de esforço computacional e acréscimo de complexidade ao modelo.

O planejamento de cobertura de RFU é, naturalmente, um problema de otimização multiobjetivo, e por isso é conveniente fornecer ao projetista do sistema as curvas de compromisso para a cobertura da área de serviço, o custo de implementação de rede, interferência interna e externa, entre outros.

Neste trabalho, é proposta uma nova abordagem baseada na avaliação multiobjetivo e multirestrição das soluções candidatas. Algumas metas, por exemplo, custo e cobertura, podem ser vistas como funções-objetivo e outras, por exemplo, exposição e interferência, podem ser tratadas como funções de restrição apresentadas para minimizar suas respectivas violações. Tal abordagem é capaz de orientar adequadamente o processo de pesquisa ao longo das melhores curvas de compromisso entre restrições e objetivos, que geralmente são conflitantes e incomensuráveis.

Esse artigo é organizado como segue: a seção II apresenta o modelo de representação de uma RFU e métricas associadas; na seção III, a abordagem multiobjetivo e multirestrição proposta e o algoritmo de otimização são apresentados; a seção IV explora o emprego da abordagem proposta e, finalmente, a seção V sumariza as conclusões. 


\section{MODELO DE REDES DE FREQUÊNCIA ÚNICA}

\subsection{Representação RFU}

O modelo de simulação de RFU empregado neste trabalho é composto por uma área de serviço, os receptores $(R)$, transmissores ( $T$ ), transmissores interferentes externos ( $T^{e x}$ ), e receptores externos interferidos ( $R^{e x}$ ). Os receptores são tomados com distribuição uniforme ao longo da área de serviço, com um espaçamento mínimo de $\Delta s$. As antenas transmissoras são tri-setorizadas a fim de moldar adequadamente a área de cobertura e evitar interferências, tanto quanto possível.

Para representar um cenário específico, os seguintes parâmetros devem ser especificados: o mapa de altimetria do terreno, ganhos de antenas transmissoras e receptoras, atenuação de setores, potência de transmissão, azimutes das antenas, frequência central do canal de transmissão e o tipo de área de serviço (urbana, suburbana ou aberta). Neste trabalho, o modelo Okumura-Hata (Hata, 1980) juntamente com o modelo de obstrução Deygout (Deygout, 1966) foram adotados para estimar as perdas de propagação. Em (Roig et al., 2010), demonstra-se que esses métodos de estimativa apresentam boa correspondência com medidas reais.

Para cada transmissor $T i \in\left\{T_{1}, T_{2}, \ldots, T_{N t}\right\}$, os seguintes parâmetros são definidos:

- $s_{i}^{a}, s_{i}^{b}, s_{i}^{c} \in\{0,1\}$ : três parâmetros do tipo ativo/inativo, indicando se os setores $a, b$ e $c$ estão ativos;

- $a_{i}^{a}, a_{i}^{b}, a_{i}^{c} \in A$ : atenuações aplicadas aos setores $a, b$ e $c$, dado um conjunto de atenuações possíveis $A$;

- $h_{i} \in H$ : altura da antena, dado um conjunto de Alturas possíveis $H$;

- $p_{i} \in P$ : potência isotrópica efetivamente irradiada, dado um conjunto $P$ de possíveis intensidades;

- $\left(x_{i}, y_{i}\right)$ : localização do transmissor, dada por índices discretos sobre a representação matricial do terreno;

- $\Theta \in\left[-90^{0}, 90^{0}\right]$ : azimute do transmissor, em que, considerando $\Theta=0$, a abertura dos setores $a, b$ e $c$ são $\left(-30^{0}, 90^{0}\right],\left(90^{\circ},-150^{\circ}\right]$, e $\left(-150^{\circ},-30^{\circ}\right]$, respectivamente.

Para cada transmissor externo, $T_{i}^{e x} \in\left\{T_{1}^{e x}, T_{2}^{e x}, \ldots, T_{N t e}^{e x}\right\}$, os seguintes parâmetros são requeridos:

- $p_{i}^{e x}$ : potência isotrópica efetivamente irradiada;

- $h_{i}^{e x}$ : altura da antena;

- $\left(x_{i}, y_{i}\right)$ : localização do transmissor externo;

Para cada receptor, $R_{i} \in\left\{R_{1}, R_{2}, \ldots, R_{N r}\right\}$, ou receptor externo interferido, $R_{i}^{e x} \in\left\{R_{1}^{e x}, R_{2}^{e x}, \ldots, R_{N r e}^{e x}\right\}$, os seguintes parâmetros devem ser especificados:

- $\left(x_{i}, y_{i}\right)$ : localização do receptor;

- $h_{R}=h_{R^{e x}}$ : altura do receptor;

- Ganho de antena, que é definido como $10 d B i$ nesse trabalho.

\subsection{Cenário de avaliação do modelo RFU}

Tomando os parâmetros de transmissores e receptores, além das informações do terreno, a relação sinal-interferência $(\Gamma)$, a probabilidade de cobertura $\left(P_{c}\right)$, os níveis de potência interferente $\left(P_{i n t}\right)$, e a exposição à emissão $\left(P_{\text {exp }}\right)$ podem ser calculadas como segue.

A "Eq. 1" fornece a relação sinal-interferência normalmente utilizada em modelos RFU (Rebhan et al., 1989; Koutitas., 2010; Ligeti et al., 1999; Ligeti, 1999).

$$
\Gamma=\frac{U}{I+N_{0}}=\frac{\sum_{i=1}^{N_{t}} U_{i}}{\sum_{i=1}^{N_{t}} I_{i}+\sum_{i=1}^{N_{t e}} P_{i}^{e x}+N_{0}}
$$

em que $N_{0}$ é a intensidade de ruído de fundo. $U_{i}$ e $I_{i}$ são, respectivamente, os componentes construtivos e destrutivos da RFU a ser avaliada, os quais podem ser calculados utilizando o modelo dado pela "Eq. 2" e "Eq. 3" , admitindo que o receptor seja sincronizado com o primeiro sinal recebido no momento $t_{0}$. $P_{i}^{e x}$ é a contribuição do i-ésimo transmissor interferente externo operando na mesma freqüência.

$$
\begin{gathered}
U_{i}=w\left(t_{i}-t_{0}\right) \cdot P_{i} \\
I_{i}=\left[1-w\left(t_{i}-t_{0}\right)\right] \cdot P_{i}
\end{gathered}
$$

em que $P_{i}$ a potência recebida do $i$-ésimo transmissor da RFU e

$$
w\left(\Delta_{t}\right)= \begin{cases}1 & , 0 \leq \Delta_{t}<T_{g} \\ \frac{T_{u}-\Delta_{t}+T_{g}}{T_{u}} & , T_{g} \leq \Delta_{t}<T_{f} \\ 0 & , \Delta_{t} \geq T_{f}\end{cases}
$$

dado que o período de símbolo $T_{f}=T_{g}+T_{u}$ é a soma do intervalo de guarda, $T_{g}$, e a parte útil, $T_{u}$, do símbolo OFDM (Rebhan and Zander, 1989).

Seguindo o modelo k-LNM como em (Lanza et al., 2012; Santella et al., 2004), a probabilidade de cobertura é calculada assumindo que as variações de potências incidentes recebidas têm distribuição log-normal, com valores médios 
representados pelos níveis de energia dados em "Eq. 1" e desvios-padrão pré-definidos, neste trabalho assumidos como $5.5 d B$ para todos os níveis de potência recebidos. Assim, a probabilidade de cobertura numa dada posição é calculada de acordo com

$$
p_{c}=P_{r}\left(\Gamma>\Psi_{0}\right)
$$

ou

$$
p_{c}=P_{r}\left(\frac{U}{I}>\Psi_{0}\right) \cdot P_{r}\left(\frac{U}{N_{0}}>\Psi_{0}\right)
$$

adotando o ruído e a interferência como variáveis aleatórias estatisticamente independentes (Rebhan et al., 1989) e fornecendo o limiar de aceitabilidade $\Psi_{0}$.

A intensidade de potência $P_{\text {int }}$ que chega a partir do transmissor $T$ e alcança um receptor externo interferido $R^{e x}$ é dada por:

$$
P_{\text {int }}=\sum_{i=1}^{N_{t}} P_{i}
$$

e não deve exceder o limiar $\Omega_{0}$.

No Brasil, o nível de densidade de potência de referência para a exposição pública geral, tratando-se de campos eletromagnéticos variantes no tempo é de $\frac{f}{200} W \cdot m^{2}$, em que $f$ é dada em $M H z$ (Anatel, 2002). Assim, a potência total medida nos receptores $R$ tomando todos os transmissores $T$, é dada por:

$$
P_{\text {exp }}=\sum_{i=1}^{N_{t}} P_{i}
$$

que não deve exceder o limiar de $\frac{f}{200}$.

\subsection{Otimização Multiobjetivo e Multirestrição}

\subsubsection{Representação}

Tipicamente, problemas de otimização do mundo real podem ser representados por um conjunto de equações com variadas restrições e objetivos (Ricny., 2007; Brownlee, 2012; Michalewicz et al., 2010; Martins, 2012; Kumar, 2008), como em

$$
\begin{array}{crr}
\text { minimizar } & \mathbf{y}= & {\left[y_{1}(x), y_{2}(x), \ldots, y_{m}(x)\right] \in Y} \\
\text { sujeito a } & \mathbf{x}= & {\left[x_{1}, x_{2}, \ldots, x_{n}\right] \in X} \\
& \mathbf{g}= & {\left[g_{1}(x, y), g_{2}(x, y), \ldots, g_{p}(x, y)\right] \leq 0}
\end{array}
$$

em que $Y \in \mathbb{R}^{m}$ é o espaço de funções-objetivo e $X \in \mathbb{R}^{m}$ é o espaço de variáveis de decisão. A notação $\mathbf{g} \leq 0$ é utilizada para fins de simplicidade, mas é fácil estendê-la a outras desigualdades e igualdades.

A fim de se avaliar numericamente a quantidade de restrições violadas, é proposta a seguinte representação alternativa de (Küchen et al., 1996):

em que

$$
\begin{array}{llr}
\text { minimizar } & \mathbf{y}= & {\left[y_{1}(x), y_{2}(x), \ldots, y_{m}(x)\right] \in Y} \\
\text { sujeito a } & \mathbf{x}= & {\left[x_{1}, x_{2}, \ldots, x_{n}\right] \in X \mathrm{e}} \\
\text { aplicado a } & \mathbf{h}= & {\left[h_{1}\left(g_{1}\right), h_{2}\left(g_{2}\right), \ldots, h_{p}\left(g_{p}\right)\right]}
\end{array}
$$

$$
h_{i}\left(g_{i}\right)=\left\{\begin{array}{ll}
-\varepsilon & , g_{i} \leq 0 ; \\
g_{i} & , g_{i}>0 .
\end{array} \quad, i=1,2, \ldots, p\right.
$$

Para a $i$-ésima violação de restrição, $g_{i}$ é a medida quantitativa da violação. Não havendo violação, a função de restrição é definida com um valor negativo infinitesimal $-\varepsilon$. O fato de $\varepsilon$ ser negativo representa a não violação de qualquer restrição. A dimensão infinitesimal consta apenas como formalidade de representação, uma vez que qualquer valor negativo poderia ser empregado.

Em Santella et al. (2004), a minimização de h é preferida quando comparada à minimização de $\mathbf{y}$, ou seja, a não violação das restrições precede a otimização das funções-objetivo. Assim, pode ser vista como uma representação de restrições severas. Por outro lado, em uma representação de restrições brandas não há prioridade entre a otimização de objetivos e funções de restrição. 


\subsubsection{Avaliação com restrições severas}

Nesse trabalho usa-se da representação com restrições severas (Santella et al., 2004). Para tanto, consideremos duas soluções candidatas $x^{a}$ e $x^{b}$ que tenham sido previamente avaliadas pela função objetivo e de restrição, dando $\left(y^{a}, h^{a}\right)$ e $\left(y^{b}, h^{b}\right)$, respectivamente. Em um processo de otimização, a fim de classificar soluções candidatas atuais é adequado compará-las aos pares, aplicando o conceito de dominância de Pareto (Ricny., 2007; Brownlee, 2012; Michalewicz et al., 2010), dado que o problema conta com mais de um critério de avaliação. A solução $x^{a}$ é melhor, ou domina, $x^{b}$ se: $(i)$ $x^{a}$ não viola qualquer restrição e $x^{b}$ viola; ou $(i i)$ as duas candidatas não violam qualquer restrição e

$$
\begin{aligned}
& \forall_{i} \in\{1,2, \ldots, m\}: y_{i}^{a} \leq y_{i}^{b} \\
& \mathrm{e} \\
& \exists j \in\{1,2, \ldots, m\}: y_{j}^{a} \leq y_{j}^{b}
\end{aligned}
$$

ou (iii) ambas as candidatas violam uma ou mais restrições e

$$
\begin{aligned}
& \forall_{i} \in\{1,2, \ldots, p\}: h_{i}^{a} \leq h_{i}^{b} \\
& \mathrm{e} \\
& \exists j \in\{1,2, \ldots, p\}: h_{j}^{a} \leq h_{j}^{b}
\end{aligned}
$$

A "Figura 1" ilustra a avaliação com restrições severas, sendo que, neste caso, as restrições são expressas como uma região factível dentro do espaço de objetivos. Essa representação acelera o processo de busca dentro do espaço de soluções factíveis, já que avalia melhor pontos que não violam restrições e pontos mais próximos da região factível.

O método de classificação de soluções acima indicado considera um problema de minimização, mas ele pode ser facilmente convertido para um problema de maximização, uma vez que a minimização de $\varphi(x)$ é equivalente à maximização de $-\varphi(x)$ e vice versa.

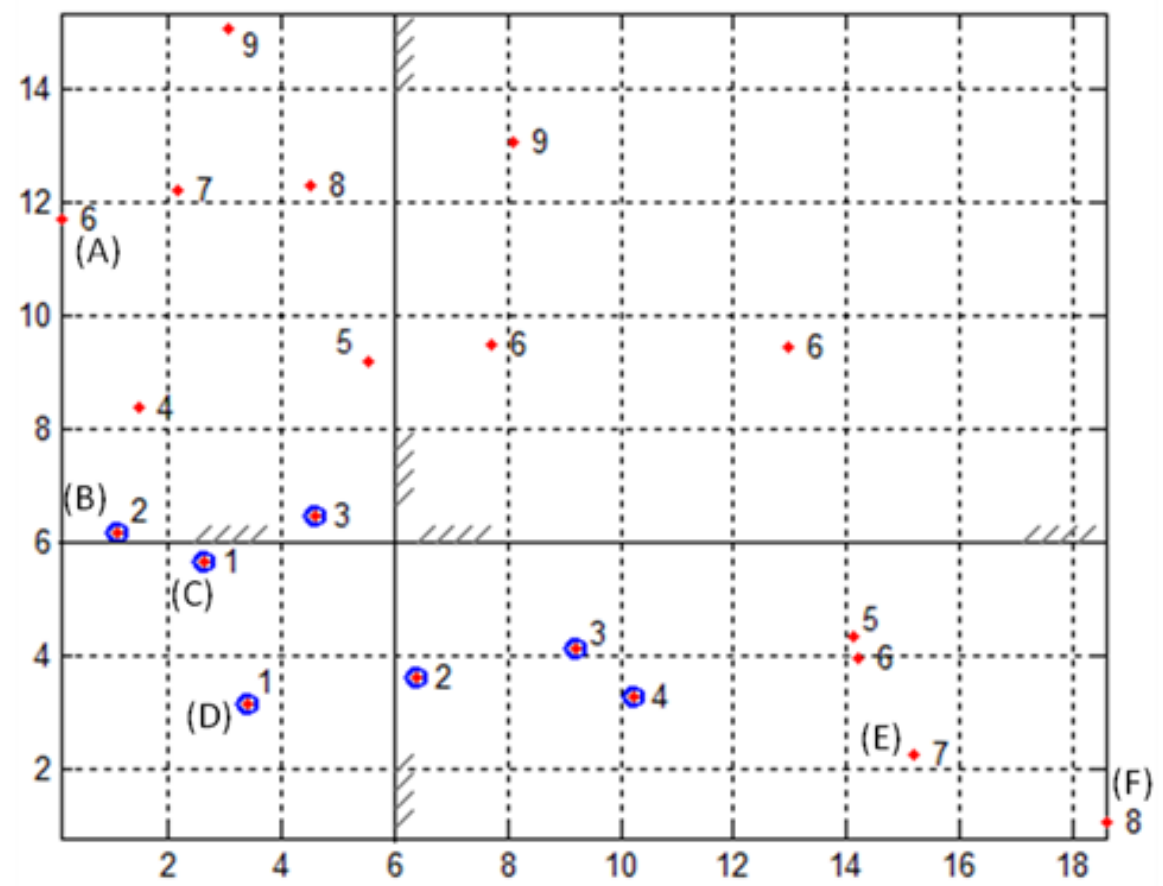

Figura 1. Classificação com restrições severas. As linhas grifadas demarcam a região factível. Os números indicam a que frente pertencem os pontos.

\subsubsection{Algoritmo de Otimização}

Um algoritmo de otimização simples (busca direta) é utilizado em conjunto com o método de avaliação descrito na seção anterior. A simplicidade no algoritmo foi adotada intencionalmente para mostrar a capacidade inerente de classificação de dominância multi-critérios para fornecer naturalmente diversificação entre as soluções ao longo do processo de busca, evitando a estagnação em ótimos locais (Brownlee, 2012; Michalewicz et al., 2010). 


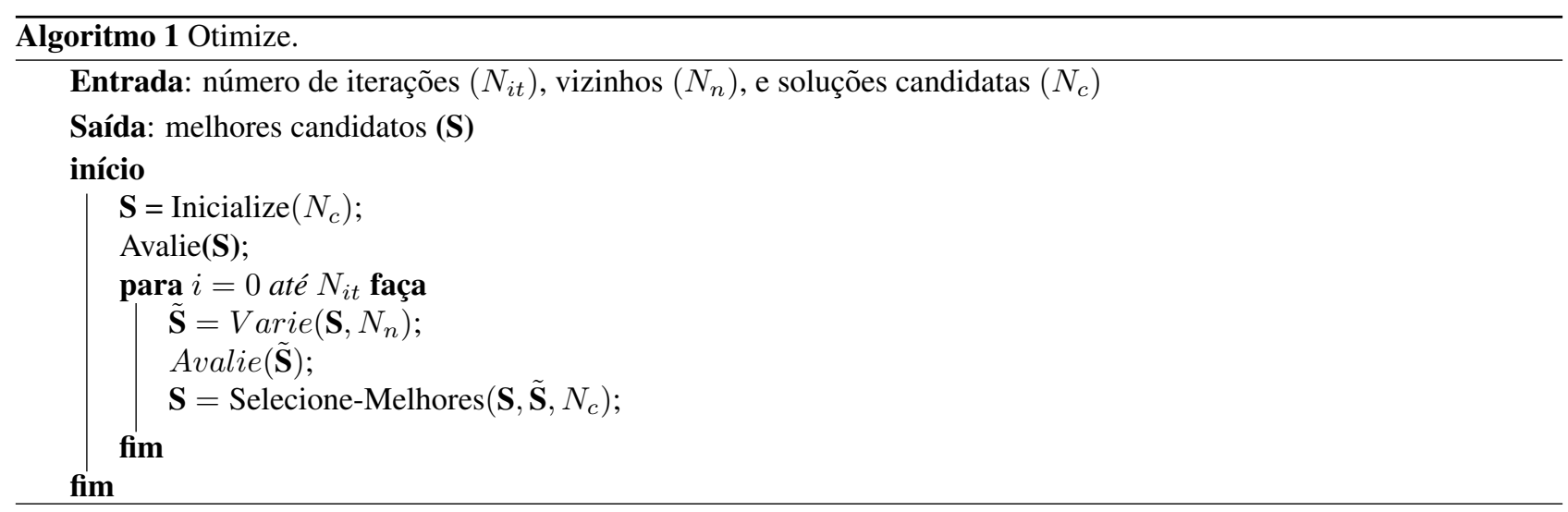

\section{Inicialização}

Como primeiro passo da otimização, na função Inicialize, o primeiro conjunto de soluções candidatas $\mathbf{S}$ é inicializado aleatoriamente. Cada solução candidata é composta por $N_{t}$ subconjuntos contendo os parâmetros de cada transmissor $T_{i} \in\left\{T_{1}, T_{2}, \ldots, T_{n t}\right\}$, ou seja, os subconjuntos fornecem os valores de $\left(s_{i}^{a}, s_{i}^{b}, s_{i}^{c}\right),\left(a_{i}^{a}, a_{i}^{b}, a_{i}^{c}\right), h_{i}, p_{i}$, e $\Theta$ para cada $T_{i}$.

\section{Avaliação}

A avaliação multicritério de uma solução candidata, dado pela função Avalie, é realizada primeiramente com o cálculo da probabilidade de cobertura usando "Eq. 6", a potência interferente usando "Eq. 7" , e a relativa exposição à radiação eletromagnética usando a "Eq. 8". Posteriormente, as quatro funções de avaliação são calculadas como se segue:

(a) Cobertura - A cobertura, $C_{\%}$, é a proporção de pontos receptores $\mathbf{R}$ que tem uma probabilidade de cobertura maior que um limiar $\left(p_{c}>\Psi_{0}\right.$, com $\left.\max C_{\%}=1\right)$.

(b) Proteção - A aqui chamada proteção, $I_{\%}$ é a proporção de pontos receptores externos $\mathbf{R}^{e x}$ que não tem o limiar $\Omega_{0}$ de máxima interferência excedido $\left(P_{\text {int }}<\Omega_{0}\right.$, com $\left.\max I_{\%}=1\right)$.

(c) Exposição - A exposição, $E_{\%}$ é a proporção de pontos de recepção $\mathbf{R}$ que não tem o limiar de máxima exposição à radiação excedido $\left(P_{\text {exp }}<\frac{f}{200}\right.$, com $\left.\max E_{\%}=1\right)$.

(d) Custo - Finalmente, a quarta função de avaliação, $C_{o}$, é o custo estimado de infraestrutura para a RFU proposta.

Portanto, o conjunto de critérios disponíveis utilizado no modelo de otimização proposto é definido como $\left(C_{\%}, I_{\%}, E_{\%}, C_{0}\right)$.

A fim de selecionar as melhores candidatas em cada iteração do algoritmo de otimização com abordagem de restrições severas, em "Eq. 10" pode ser usada, definindo:

$$
\boldsymbol{y}=\left[\left(1-C_{\%}\right), C_{o}\right] \quad \text { e } \quad \boldsymbol{g}=\left[\left(1-I_{\%}\right),\left(1-E_{\%}\right)\right]
$$

que representa uma otimização multiobjetivo $y$ e multirestrição $g$. Por conseguinte, nesse caso, é desejável minimizar o custo e maximizar a área de cobertura, além da maximização da proteção contra interferências e a minimização da exposição à radiação.

Na otimização com restrições brandas, vista em "Eq. 12", pode-se definir:

$$
\boldsymbol{f}=\left[\left(1-C_{\%}\right),\left(1-I_{\%}\right),\left(1-E_{\%}\right), C_{o}\right]
$$

que tipifica um problema de otimização multiobjetivo puro.

\section{Variação}

Cada solução candidata é variada ao selecionar e alterar um de seus parâmetros, atribuindo aleatoriamente um dos possíveis valores de seu respectivo conjunto, com exceção do valor de azimute, para o qual o novo valor é fornecido de acordo com a "Eq. 16"

$$
\tilde{\Theta}=\Theta+\sigma \cdot N(0,1)
$$

em que $\Theta$ é o azimute corrente, e o $\Theta$ é o novo valor, $\sigma$ é o desvio, e $N(0,1)$ representa uma distribuição de probabilidade Gaussiana com média zero e desvio unitário. Empiricamente, o valor mais adequado encontrado para $\sigma$ foi $5^{0}$.

A cada iteração do otimizador, $N_{n}$ vizinhos de cada $N_{c}$ solução candidata corrente são gerados, o que provê $(1+$ $\left.N_{n}\right) \cdot N_{c}$ candidatas para serem avaliadas e comparadas. 


\section{Seleção}

Ao longo da otimização, uma vez que o número corrente de soluções candidatas é limitado por $N_{c}$, é necessário truncar o conjunto de candidatas avaliadas $\left(1+N_{n}\right) \cdot N_{c}$ a cada iteração. A seleção das melhores candidatas é realizada seguindo os passos:

(a) Iterativamente classifica-se o conjunto $\left(1+N_{n}\right) \cdot N_{c}$ candidatas usando o conceito de dominância de Pareto. Na primeira iteração $(i=1)$, as soluções candidatas não-dominadas são rotulados como pertencentes à primeira frente de Pareto (melhores soluções). Então, de forma incremental, na $i$-ésima iteração, ignorando as soluções já rotuladas, as candidatas não-dominadas são então rotuladas como pertencentes ao $i$-ésima frente. Esse processo é repetido até que o número de candidatas rotuladas seja maior ou igual a $N_{c}$. Todas as soluções não rotuladas são então eliminadas do conjunto inicial;

(b) Na sequência, as candidatas remanescentes com avaliações inferiores, rotuladas em frentes de maior ordem, são tomadas para nova poda. Iterativamente, uma candidata que tem a mínima distância média para seus $k$ vizinhos mais próximos é eliminada a cada passo (método $k$-nearest-neighbors). Esse passo é repetido enquanto o tamanho do conjunto é maior que $N_{c}$.

O método k-nearest-neighbors (Silverman, 1986) é usado neste trabalho, devido à sua simplicidade e aos bons resultados apresentados para a estimativa da densidade de soluções dentro do espaço de busca. O valor $k$ é determinado pelo número inteiro mais próximo da raiz quadrada do número de candidatas a serem comparadas em cada etapa. Além disso, antes da aplicação desse método, cada função de avaliação (função de restrição ou objetivo) é normalizada dimensionandoa entre 0 e 1 .

O método proposto de poda é capaz de preservar as candidatas com os valores de função de avaliação extremas e sustenta uma distribuição aproximadamente uniforme ao longo da melhor frente (primeira), tal como ilustrado na "Fig. 2"

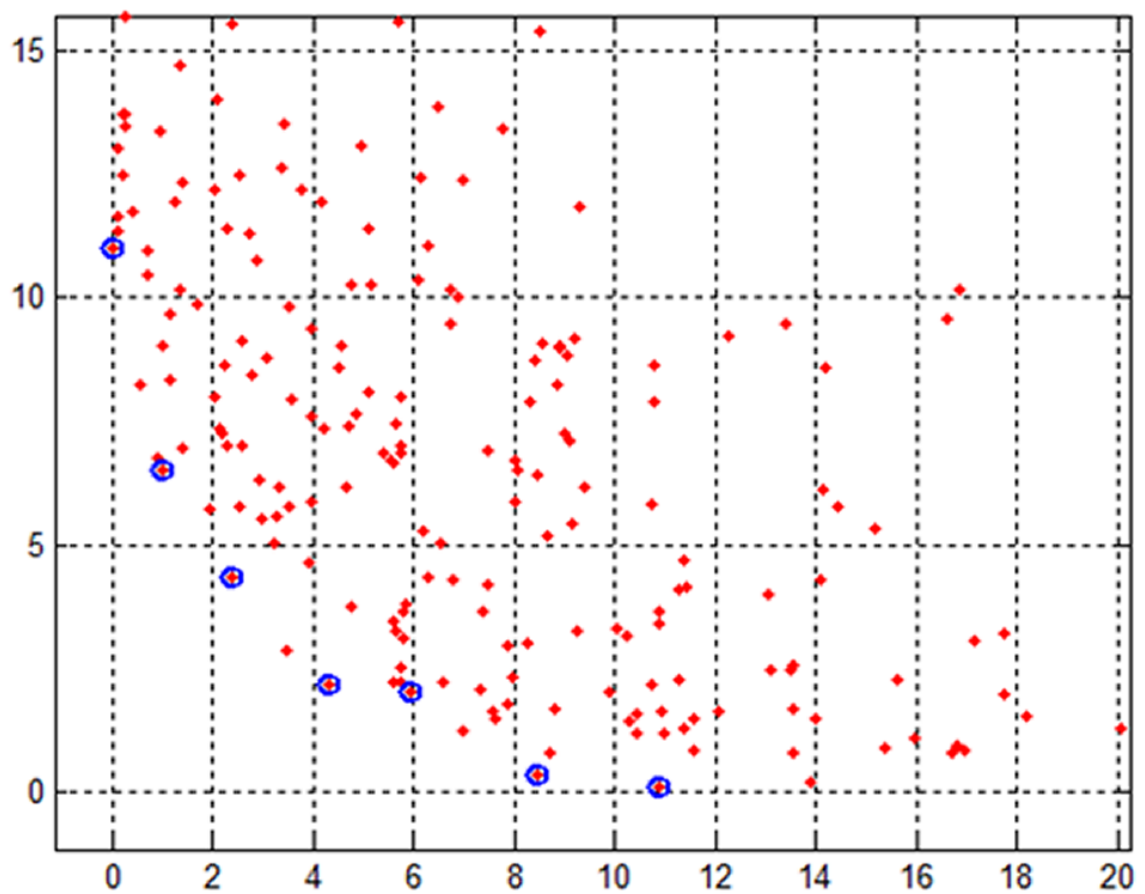

Figura 2. Ilustração do método de poda. Os círculos indicam as sete melhores soluções, considerando duas funções objetivo arbitrárias.

\section{ESTUDO DE CASO}

O estudo de caso usa os parâmetros de inicialização definidos na "Tab. 1" e os parâmetros de otimização mostrados na "Tab. 2"

Todas as otimizações realizadas utilizaram número de iterações $N_{i t}=1000, N_{c}=30$, e $N_{n}=1$. A "Fig. 3" mostra o mapa de altimetria usada para o estudo de caso. O contorno da área de serviço (polígono interno) delimita as possíveis localizações dos transmissores. O contorno externo contém os receptores externos. Este cenário ilustra um planejamento de RFU não interferente de um modo extremo, completamente circundado, uma vez que se faz necessário que o sinal fique confinado dentro da área de serviço. 
Tabela 1. Parâmetros de Inicialização

\begin{tabular}{c|c|c}
\hline Símbolo & Parâmetro & valor \\
\hline $\boldsymbol{f}$ & Frequência do canal & $472 \mathrm{MH}$ \\
\hline$\Psi_{0}$ & Minima relação sinal-interferência & $17 \mathrm{~dB}$ \\
\hline$\Omega_{0}$ & Limiar de Proteção & $-65 \mathrm{dBW}$ \\
\hline$T_{g}$ & Tempo de guarda do símbolo OFDM & $224 \mathrm{\mu s}$ \\
\hline$T_{u}$ & Tempo útil do símbolo OFDM & $896 \mu \mathrm{s}$ \\
\hline$N_{0}$ & Potência do ruído de fundo & $-60 \mathrm{dBW}$ \\
\hline$h_{R}, h_{R^{e x}}$ & Altura da antena receptora & $5 \mathrm{~m}$ \\
\hline$G_{R x}$ & Ganho da antena receptora & $10 \mathrm{dBi}$ \\
\hline$G_{T x}$ & Ganho da antena de transmissão & $20 \mathrm{dBi}$ \\
\hline
\end{tabular}

Tabela 2. Parâmetros de Otimização

\begin{tabular}{c|c|c|c}
\hline Símbolo & Parâmetro & Valores Possíveis & Custo normalizado \\
\hline $\boldsymbol{A}$ & Atenuação por setor de antena & $\{0 ; 3 ; 6\} \mathrm{dB}$ & despresível \\
\hline $\boldsymbol{H}$ & Altura de antena & $\{20 ; 30 ; 40 ; 60\} \mathrm{m}$ & $\{160 ; 180 ; 150 ; 350\}$ \\
\hline $\boldsymbol{P}$ & Potência efetivamente irradiada & $\{20 ; 30 ; 40 ;\} \mathrm{dBW}$ & $\{50 ; 100 ; 150\}$ \\
\hline
\end{tabular}

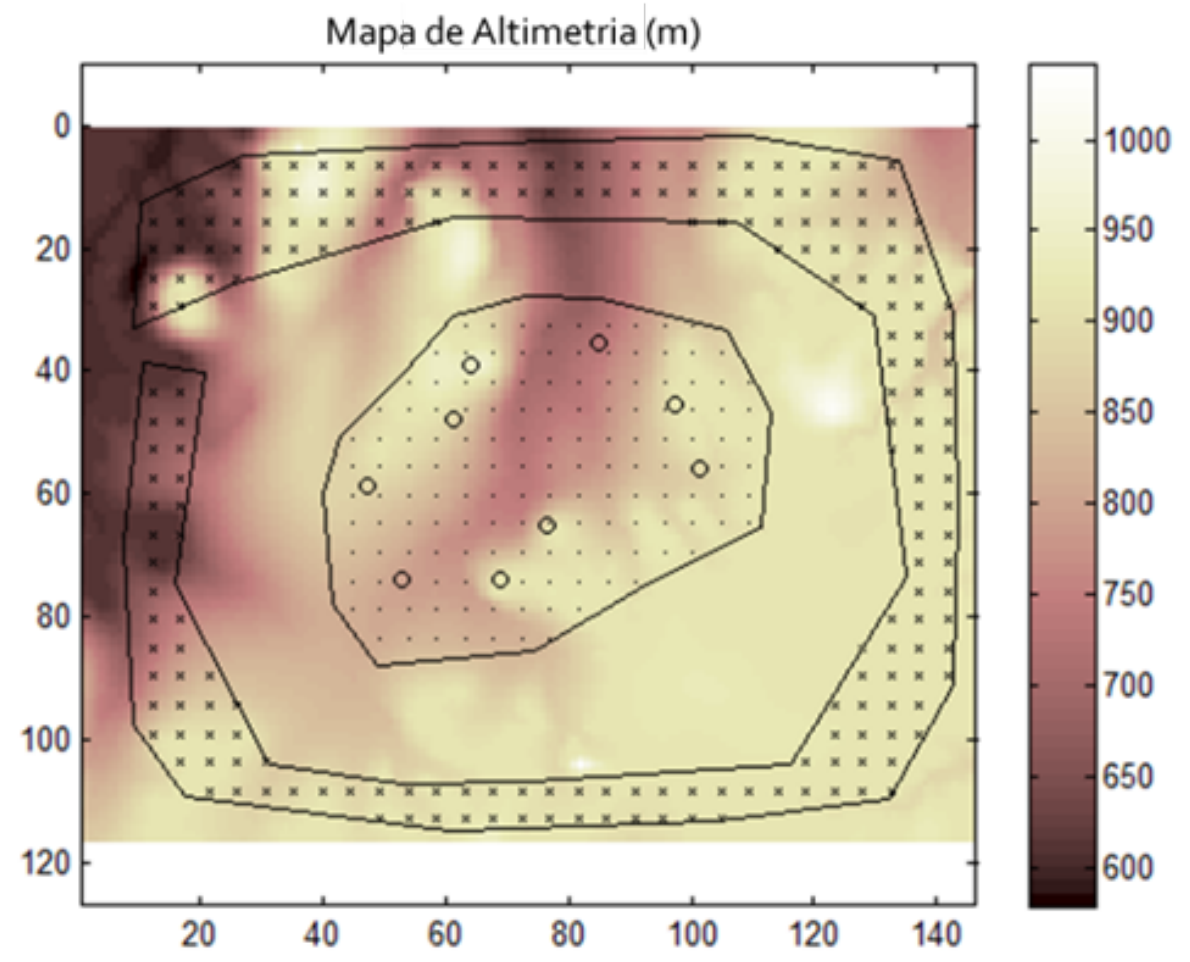

Figura 3. Mapa de altimetria. Os círculos confinados na parte central são as possíveis localizações das antenas de transmissão.

\subsection{Otimização de custos e cobertura como objetivos, proteção e exposição como restrições}

A cobertura e os custos são tratados como objetivos e a proteção e a exposição são tomadas como restrições do problema de otimização. A "Fig. 4" mostra as soluções obtidas. Cabe ressaltar que todas as soluções alcançadas, além de respeitarem o limiar de interferência "Fig. 6" não violam as restrições referentes aos limiares de proteção e exposição eletromagnética, o que evidencia a eficácia da abordagem. As "Fig. 5" e "Fig. 6" mostram os resultados correspondentes a uma das melhores soluções, a qual apresenta um percentual de cobertura maior que $85 \%$ a um custo normalizado de 1410 unidades. 

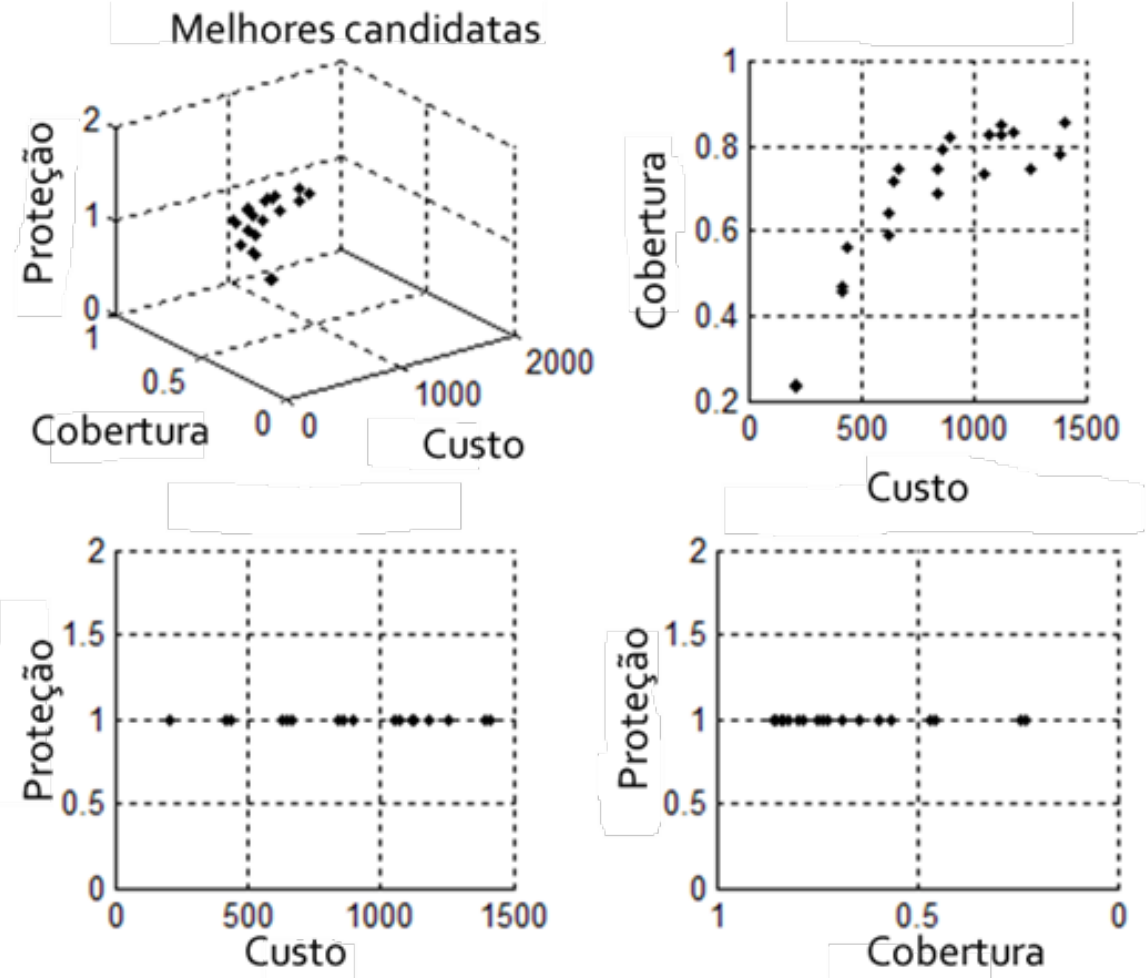

Figura 4. Melhores soluções candidatas encontradas dentro do espaço de busca.

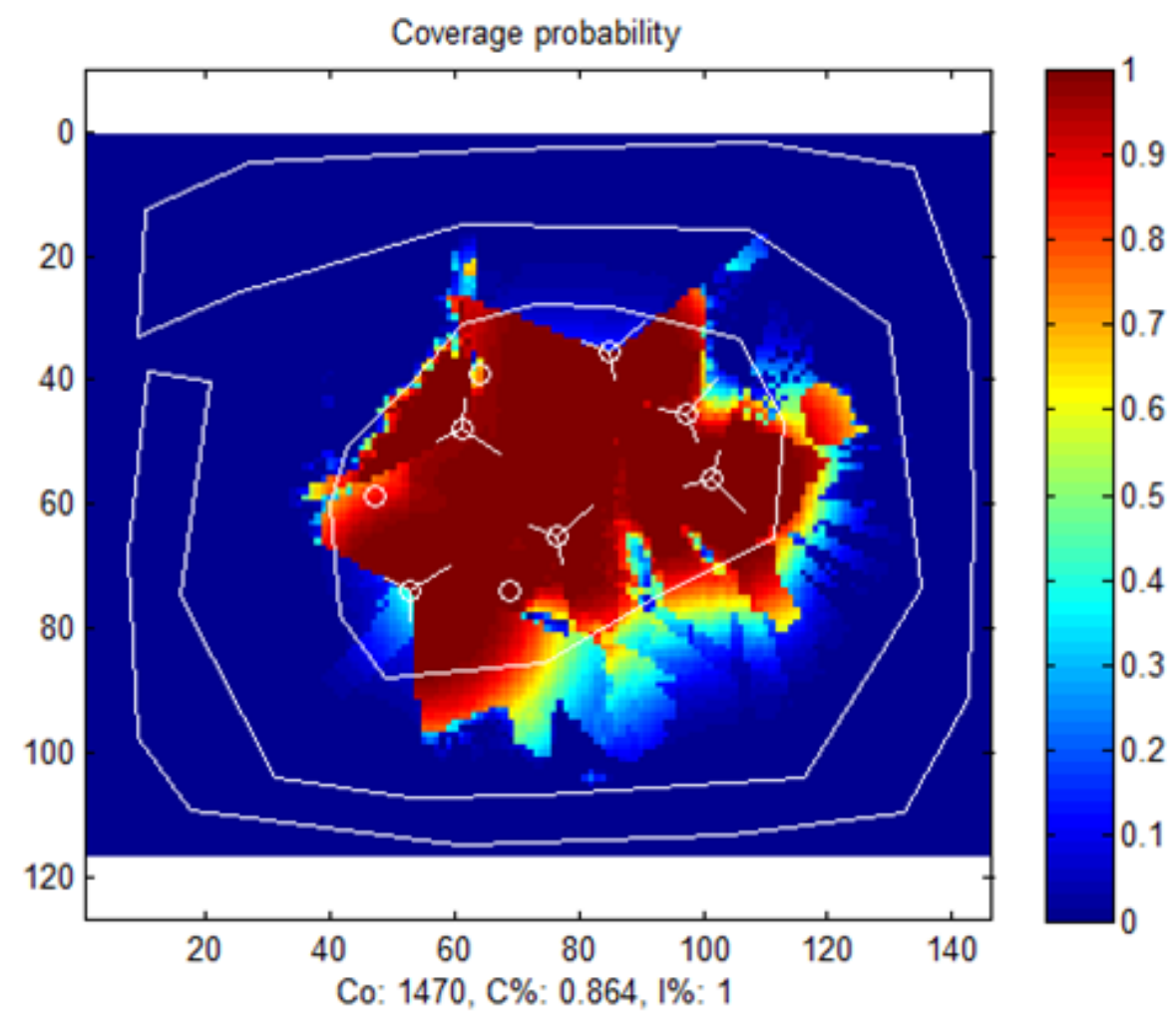

Figura 5. Probabilidade de cobertura dentro da área de serviço. 


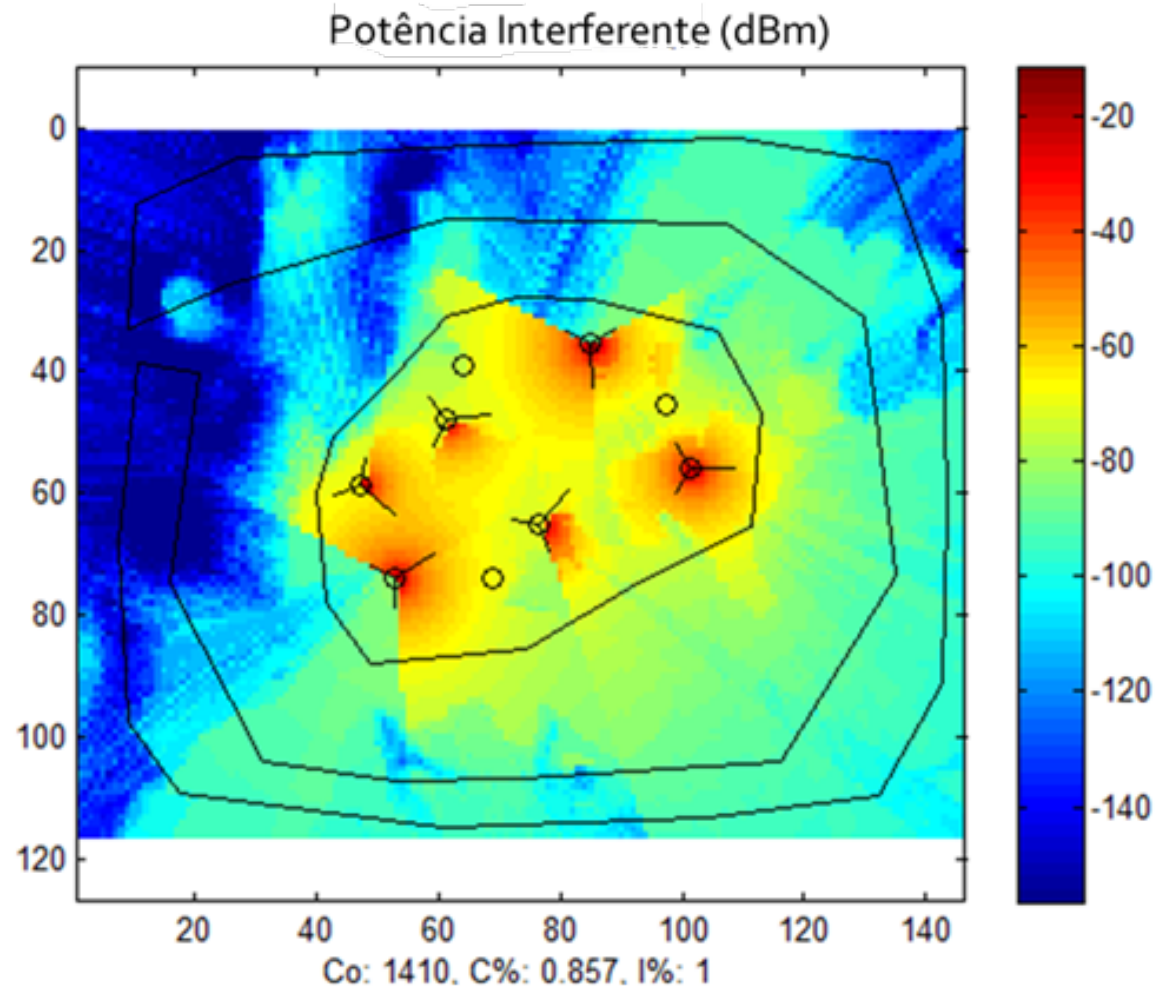

Figura 6. Intensidade de potência interferente.

\section{CONCLUSÃO}

Uma vez que existem diversas variáveis de decisão e funções de avaliação lineares e não-lineares envolvidas no planejamento de RFU, se fez necessário um modelo de simulação combinatorial para avaliar cenários de otimização. Embora o referido problema seja tipicamente representado na literatura usando uma única função de avaliação (monoobjetivo), a característica inerente a tal classe de problemas de otimização, seu caráter multiobjetivo e multirestrição, motivou o uso de uma abordagem com múltiplos critérios para representar e avaliar melhor soluções candidatas. A fim de contribuir neste campo, o presente trabalho propôs um método de otimização que oferece soluções otimizadas para o problema de cobertura de RFU. Aqui, o modelo com quatro funções de avaliação foi tratado com a representação multicritério, podendo ser estendido para abranger outros aspectos, tais como a maximização do consumo de energia e minimização das emissões de carbono (Hata, 1980)além de poder ser aplicado a problemas de cobertura no âmbito de outros sistemas, tais como Wi-Fi, WiMAX, redes celulares, e outros. A inovação do modelo baseia-se na melhoria na forma de se avaliar a qualidade das soluções considerando também a distribuição dentro do espaço de busca. Comparações no que se refere à performance e consumo computacional podem ser aferidas de acordo com (Rocha et al., 2012).

\section{REFERÊNCIAS}

R. Rebhan and J. Zander On the outage probability in Single Frequency Networks for digital broadcasting. IEEE Trans. on Broadcasting, vol. 39, n.4, (pp. 395-401). Dec, 1993.

M.M. Vélez, P. Angueira, D. De la Vega, et al. Measurement based software design for DVB-T an T-DAB Single Frequency Network planning and coverage prediction Proc. 5th Int. Sym. on Antennas, Propagation and EM Theory, (pp. 607-610). 2000.

D. Plets, W. Joseph, P. Angueira, et al. On the methodology for calculating SFN gain in digital broadcast systems IEEE Trans. on Broadcasting, vol. 56, n.3, (pp. 331-339). Sep, 2010.

G. Koutitas. Green network planning of Single Frequency Networks IEEE Trans. on Broadcasting, vol. 56, n.4, (pp. 541-550). Dec, 2010.

M. Lanza, A.L. Gutiérrez, I. Barriuso, et al. Coverage optimization and power reduction in SFN using a hybrid PSO algorithm Proc. 6th European Conference on Antennas and Propagation (EUCAP), (pp. 2043-2047). Prague 2012.

M. Lanza, A.L. Gutiérrez, I. Barriuso, et al. Coverage optimization in Single Frequency Networks using Simulated Annealing Proc. Int. Sym. on Antennas and Propagation (APSURSI), , (pp. 2789-2792). Santander, Jul. 2011.

A. Ligeti and J. Zander. Minimal cost coverage planning for Single Frequency Networks IEEE Trans. on Broadcasting, , Vol. 45, n. 1 (pp. 78-87). Mar. 1999.

A. Ligeti. Single Frequency Network planning Radio Comm. Sys. Lab, Royal Inst. of Tech, Ph.D. thesis. Stockholm, Sweden, 1999. 
F. Küchen, T.C. Becker, and W. Wiesbeck. Optimizing the coverage area of Single Frequency Networks Proc. Int. Broadcasting Convention, (pp. 236-241). Amsterdam 1996.

G. Santella, R. Martino, and M. Ricchiuti. Single Frequency Network (SFN) planning for digital terrestrial television and radio broadcast services: the Italian frequency plan for T-DAB Proc. Vehicular Technology Conference (VTC), (pp. 2307-2311). Naples 2004.

V. Ricny. Single Frequency Networks (SFN) in digital television broadcasting Radioengineering, vol. 16, n. 4 (pp. 2-6). Dec. 2007.

K. Deb. Multi-Objective Optimization using Evolutionary Algorithms John Wiley \& Sons, Inc, New York, NY, USA, 2001.

K. Miettinen. Nonlinear Multiobjective Optimization Kluwer Academic Publishers, International Series in Operations Research \& Management Science, vol. 12, 1999.

M. Hata. Empirical formula for propagation loss in land mobile radio services IEEE Trans. Veh. Tech, vol. 29, n. 3, (pp. 317-325). 1980.

J. Deygout. Multiple knife-edge diffraction of microwaves IEEE Trans. on Antennas and Propagation, vol. 14, n. 4, (pp. 480-489). Jul. 1966.

G. Roig, D. Gomez-Barquero, and N. Cardona. Propagation model calibration for DVB-SH in terrestrial Single Frequency Networks Proc. 4th Int. Euro. Conf. on Antennas and Propagation, (pp. 1-5). Valencia, Apr. 2010.

National Telecommunications Agency Resolution n. 303 National Telecommunications Agency (Anatel, Brazil), Jul. 2002.

J. Brownlee. Clever rithms: nature-inspired programming recipes $L u L u, 1$ st ed., 2012.

Z. Michalewicz and D.B. Fogel. How to solve it: modern heuristics Springer, 2nd ed., 2010.

R. Martins. Multi-objective multi-constraint routing of analog ICs using a modified NSGA-II approach Proc. Int. Conf. on Synthesis, Modeling, Analysis and Simulation Methods an Applications to Circuit Design (SMACD), (pp. 65-68). Lisbon, Sep. 2012.

Y. Kumar. Multiobjective, Multiconstraint Service Restoration of Electric Power Distribution System With Priority Customers IEEE Trans. on Power Delivery, vol. 23, n. 1, (pp. 261-270). Jan. 2008.

B. W. Silverman. Density estimation for statistics and data analysis Chapman and Hall, Londres, 1986.

A. S. Rocha and C.J.A Macedo and P. H. S. Palhares and L. C. Brito. An Improved Multiobjective Search Method Applied to Single Frequency Networks Planning Latin America Transactions, IEEE (Revista IEEE America Latina), vol. 10, n. 1, (pp. 1143-1148). Jan. 2012.

\section{RESPONSABILIDADE AUTORAL}

“O(s) autor(es) é(são) o(s) único(s) responsável(is) pelo conteúdo deste trabalho”.

\section{MULTI-OBJECTIVE MULTI-CONSTRAINT COVERAGE OPTIMIZATION OF SINGLE FREQUENCY NETWORKS}

\footnotetext{
Adson Silva Rocha, adsonrocha@gmail .com ${ }^{1}$

Leonardo da Cunha Brito, brito. lc@gmail . com $^{2}$

Ciro José Almeida Macedo, ciro.macedo@ifg.edu.br ${ }^{3}$

${ }^{3}$ IF Goiano - Instituto Federal Goiano - Rua 88, no 310, Setor Sul, Goiânia - GO

${ }^{4}$ UFG - Escola de Eng. Elétrica, Mecânica e de Computação - Av. Universitária, Setor Lest. Universitário, Goiânia-GO

${ }^{5}$ IFG - Instituto Federal de Educação Ciências e Tecnologia de Goiás - Quartel do XX, Centro, Goiás-GO

\begin{abstract}
This paper presents a multi-objective multi-constraint approach for optimization of Digital Video Broadcasting $(D V B)$ systems based on Single Frequency Networks (SFN). The proposed method is capable of providing the trade-off curves of coverage, costs, and interference, besides others as exposure to radiation level. The proposed general coverage optimization method can be extended to other wireless networks, such as cellular systems.
\end{abstract}

Keywords: OFDM, Single Frequency Network, multi-objective, multi-constraint optimization 